\section{Prevalence of serum autoantibodies and paraproteins in patients with glaucoma}

\begin{abstract}
Purpose To investigate the possible link between normal tension glaucoma (NTG) and autoimmunity.

Methods We studied the serum of 95 patients: 31 with NTG, 32 with primary open-angle glaucoma (POAG), and 32 age- and sexmatched controls. Blood was drawn from each patient and serum was examined for the presence of antinuclear antigens (ANA), autoantibodies to extractable nuclear antigens (ENA), anti-double-strand DNA, serum protein electrophoresis, and immunoglobulin (IgG, IgA, and IgM) levels.

Results In the NTG group, the relative risks for ANA and ENA positivity were 2.5 and 4.4 times, respectively, that of the control group. There was a statistically significant difference between IgA levels in the NTG and control group $(P=0.024)$, but there was no statistically significant difference between both groups regarding IgM or IgG levels. In the POAG group, the relative risks for ANA and ENA positivity were 0.77 and 2.9 times, respectively, that of the control group. The relative risk for detection of paraprotein in the POAG group was 0.97 times that of the control group. Also, there was a statistically significant difference between IgA levels in the POAG and control group $(P=0.011)$, but there was no statistically significant difference between both groups regarding IgM or IgG.

Conclusion These results support the hypothesis that humoral immune mechanisms may have a role in the pathogenesis of NTG.

Eye (2008) 22, 349-353; doi:10.1038/sj.eye.6702613; published online 27 October 2006
\end{abstract}

Keywords: normal tension glaucoma; primary open-angle glaucoma; paraproteinaemia; autoantibodies
T Hammam, D Montgomery, D Morris and F Imrie

\section{Introduction}

Glaucoma is usually associated with elevated intraocular pressure (IOP). Classical theories attribute optic nerve damage from elevated IOP either to mechanical trauma at the cribriform plates or to alterations in vascular perfusion and subsequent ischaemia. ${ }^{1}$ However, some patients with visual field loss and optic disc cupping typical of glaucoma have normal IOP. These patients are classified as having normal tension glaucoma (NTG). Typical population surveys show that $10-30 \%$ of newly diagnosed glaucoma patients have NTG. ${ }^{2}$

Although the IOP in NTG lies within the normal range, there is still evidence that it is a risk factor for the development and progression of the disease. ${ }^{3}$ However, the NTG study showed that, in some patients, there was no relationship between the pressure levels and disease outcome, suggesting a disease process that is independent of IOP. ${ }^{4}$

The pathogenesis of optic neuropathy in NTG has been examined in several studies. Increased resistance in the ophthalmic and central retinal artery has been demonstrated in patients with NTG compared with controls. ${ }^{5}$ Greater nocturnal drops in both systolic and diastolic blood pressure have been found in NTG compared with normal subjects, suggesting a possible vascular mechanism. ${ }^{6,7} \mathrm{O}^{\prime}$ Brien et $a l^{8}$ found a relative activation of the coagulation cascade and fibrinolysis pathways in primary open-angle glaucoma (POAG) and NTG compared with control suggesting abnormal rheology in NTG.

Epidemiological studies have shown that patients with NTG have significantly more systemic autoimmune disorders than patients with POAG, raising the possibility of a cross reaction between antigenic stimuli related to systemic autoimmune disorder and antigens in the optic nerve head. ${ }^{9}$
Glasgow Royal Infirmary, Alexandra Parade, Glasgow, UK

Correspondence:

T Hammam, Glasgow Royal Infirmary, Alexandra Parade, Glasgow, UK Tel: + 4407790856654 ; Fax: + 4401412112054 E-mail: thammamaol.com

Received: 31 June 2006 Accepted in revised form: 8 August 2006 Published online: 27 October 2006

This work was previously presented at the Scottish Ophthalmological Club meeting October 2003 
Wax et al described an increased incidence of autoantibodies and paraproteins in NTG compared with POAG and controls. ${ }^{2}$ This study found a statistically significant increase in autoantibodies to extractable nuclear antigens (ENA) and serum monoclonal proteins in NTG, but failed to show a statistically significant increase in antinuclear antibodies. Other workers have identified immunoglobulin deposition in the retina in NTG ${ }^{10}$ whereas numerous elevated serum autoantibody titres have also been demonstrated, for example, to optic nerve head glycosaminoglycans (GAGs), ${ }^{11}$ to gammaenolase, ${ }^{12}$ to retinal antigens (including rhodopsin ${ }^{13}$ ), to heat-shock proteins, ${ }^{14}$ and to glutathione $S$-transferase. ${ }^{15}$

\section{Purpose}

To investigate the possible link between NTG and autoimmunity.

\section{Materials and methods}

Ninety-five patients were selected for this study and divided into three cohorts, matched for age and sex: 31 with NTG, 32 with POAG, and 32 healthy controls. The characteristics of each group are shown in Table 1. Patients were recruited from specialist glaucoma and general clinics. The diagnosis of POAG or NTG was made independently, without knowledge of the serum autoantibodies or immunofixation results. Inclusion criteria for patients with NTG comprised the

Table 1 Characteristics of the patients in each group

\begin{tabular}{lccc}
\hline & NTG & POAG & Control \\
\hline No. of females & 24 & 24 & 24 \\
No. of males & 7 & 8 & 8 \\
Mean age (years) & 75 & 75.5 & 75 \\
Range (years) & $51-87$ & $51-87$ & $51-88$ \\
\hline
\end{tabular}

following: a mean IOP offtreatment consistently equal to or less than $21 \mathrm{~mm} \mathrm{Hg}$ with no single measurement greater than $24 \mathrm{~mm} \mathrm{Hg}$, open angles on gonioscopy, absence of any secondary cause for a glaucomatous optic neuropathy, typical optic disc damage with glaucomatous cupping and loss of neuroretinal rim, visual field defect compatible with glaucomatous optic disc cupping. Similar inclusion criteria were applied for patients with POAG, except that the mean IOP was equal to or greater than $22 \mathrm{~mm} \mathrm{Hg}$ on presentation.

The control group was recruited from the cataract assessment clinics. Patients with other ocular or any autoimmune disease were excluded from the study. Patient consent was obtained and blood was taken and tested for the presence of antinuclear antibodies (ANA), autoantibodies to ENA (including anti-\{Ro, La, Smith(Sm), RNP(ribo-nucleo-protein), Jo, Scl $\}$, antidouble-strand DNA (anti-dsDNA), serum protein electrophoresis, and immunoglobulins (IgG, IgA, and IgM). RELISA kit (manufactured by Immunoconcept) was used for testing ENA.

\section{Results}

Table 2 shows the prevalence of positive ANA, ENA, anti-dsDNA, and paraprotein in the three groups. Ten patients $(32.3 \%)$ in the NTG group had antinuclear antibodies in their serum compared with only four $(12.5 \%)$ and five $(15.6 \%)$ patients in the POAG and control groups, respectively. Four $(12.9 \%)$ patients in the NTG group were positive for ENA compared with three $(9.37 \%)$ in the POAG group and only one $(3.12 \%)$ in the control group. Two $(6.25 \%)$ patients in the POAG group had elevated anti-dsDNA (>30 mmol/l) in their serum, compared with none in the NTG or control groups. None of the NTG patients' serum was positive for paraprotein, compared with two $(6.25 \%)$ in the POAG group and three $(9.37 \%)$ in the control group.

Table 2 Incidence of positive ANA, ENA, anti-dsDNA, and paraprotein in the three groups

\begin{tabular}{lccc}
\hline Test & NTG $\mathrm{n}=31(\%)$ & POAG $\mathrm{n}=32(\%)$ & Control $\mathrm{n}=32(\%)$ \\
\hline ANA positive & $10 / 31(32.3 \%)$ & $4 / 32(12.5 \%)$ & $5 / 32(15.6 \%)$ \\
ENA positive & $4 / 31(12.9 \%)$ & $3 / 32(9.37 \%)$ & $1 / 32(3.12 \%)$ \\
Anti-dsDNA $>30$ & $0 / 31$ & $2 / 32(6.25 \%)$ & $0 / 32$ \\
Paraprotein positive & $0 / 31$ & $2 / 32(6.25 \%)$ & $3 / 32(9.37 \%)$ \\
\hline
\end{tabular}

Table 3 Mean levels of immunoglobulins in NTG and POAG compared with controls

\begin{tabular}{llrr}
\hline & NTG & POAG & Control \\
\hline IgG level mean \pm SD (normal range $=5.3-16.5 \mathrm{~g} / \mathrm{l})$ & $9.74 \pm 2.26$ & $10.2 \pm 2.50$ & $11.28 \pm 2.53$ \\
IgA level mean \pm SD (normal range $=0.8-4.00 \mathrm{~g} / \mathrm{l})$ & $2.16 \pm 1.06$ & $2.16 \pm 0.99$ & $2.03 \pm 1.75$ \\
IgM level mean \pm SD (normal range $=0.5-2.00 \mathrm{~g} / \mathrm{l})$ & $0.94 \pm 0.61$ & $0.76 \pm 0.44$ & $0.94 \pm 0.45$ \\
\hline
\end{tabular}


Table 3 shows the mean levels of immunoglobulins in NTG and POAG compared with controls.

IgA levels showed a statistically significant difference between NTG and control groups ( $t$-test), whereas there was no statistically significant difference between these groups in respect of IgG or IgM levels (Table 4).

Similarly, there was a statistically significant difference at the 5\% level between POAG and control groups in respect of IgA level ( $t$-test), whereas there was no statistically significant difference between these groups in respect of IgG or IgM levels (Table 4).

Using Fisher's exact test, the relative risks of ANA and ENA positivity in the NTG group were 2.5 and 4.4 times, respectively, that of the control group. Neither of these was statistically significant ( $P=0.21$ and 0.33 ) (Table 5). The relative risk of anti-ds DNA could not be calculated owing to zero observations in the NTG and control groups.

In the POAG group, the relative risks of ANA and ENA positivity were 0.77 and 2.9 times, respectively, that of the control group. Again, neither of these was statistically significant $(P=0.9999$ and 0.64 ) (Table 5). The relative risk for the detection of paraprotein in the POAG

Table 4 Mean immunoglobulin levels in NTG and POAG compared with the control

\begin{tabular}{lll}
\hline & POAG & NTG \\
\hline IgG & $P=0.95$ & $P=0.17$ \\
IgA & $P=0.013^{\mathrm{a}}$ & $P=0.024^{\mathrm{a}}$ \\
IgM & $P=0.18$ & $P=0.485$ \\
\hline
\end{tabular}

aStatistically significant.

Table 5 Relative risk of positive ANA and ENA in NTG and POAG compared with the control

\begin{tabular}{lcc}
\hline & $N T G$ & $P O A G$ \\
\hline ANA & 2.5 & 0.77 \\
& $P=0.21$ & $P=0.99$ \\
ENA & 4.4 & 2.9 \\
& $P=0.33$ & $P=0.99$ \\
\hline
\end{tabular}

Table 6 Titre and pattern of positive ANA in each group

\begin{tabular}{lccc}
\hline & NTG & POAG & Control \\
\hline $\begin{array}{l}\text { Total No. of } \\
\text { positive ANA }\end{array}$ & 10 & 4 & 4 \\
Titre & $\begin{array}{c}9(1: 40) \\
\text { 1 } 1: 160)\end{array}$ & $4(1: 40)$ & $4(1: 40)$ \\
Pattern & $\begin{array}{c}\text { 9 homogenous } \\
1 \text { centromere }\end{array}$ & $\begin{array}{c}\text { 1 homogenous } \\
\text { 1 nucleolar }\end{array}$ & $\begin{array}{c}\text { 1 homogenous } \\
\text { 2 speckled }\end{array}$ \\
& & nucleolar \\
\hline
\end{tabular}

group was 0.97 times that of the control group, and this was not statistically significant $(P=0.9999)$.

Table 6 shows the titre and the pattern of the positive ANA in each group.

\section{Discussion}

IgA is the predominant immunoglobulin found in secretions such as tears and saliva, where it plays an important role in local mucosal immunity. ${ }^{16}$

The results of this study show a statistically significant difference in IgA levels between POAG and NTG groups compared with controls, but failed to demonstrate any significant difference in IgG or IgM levels. However, unlike Wax et $a l,{ }^{17}$ there was no difference in ENA positivity between the three groups. In keeping with Wax et al, we failed to demonstrate any difference in ANA positivity.

Wax et $a l^{17}$ studied the incidence of paraproteinemia and autoantibodies in NTG patients. They found that $30 \%$ of the NTG group were positive for ENA compared with $2 \%$ of patients with POAG and this difference was found to be statistically significant $(P=0.0022)$. They also found that $41 \%(18 / 44)$ of the NTG group were positive for ANA compared with $29 \%(12 / 41)$ of patients with POAG, but this difference was not statistically significant. Serum monoclonal proteins occurred in $18 \%$ of patients with NTG compared with none in the POAG and in $2 \%$ of the control group. The prevalence of paraprotein (monoclonal gammopathies) increases with age. They are present in $1-1.7 \%$ of those aged over 50 years, and occur in $3 \%$ of those older than 70 years and in nearly $6 \%$ of those in the ninth decade of life ${ }^{18}$.

In 1979, Nagasubramanian et al $^{19}$ reported that the mean levels of serum IgG were significantly higher in patients with chronic simple glaucoma than in the control group and the mean levels of IgG were similar in all grades of severity of field or disc change, but when the means were compared with the control series significant differences occurred in only the most severely affected cases. IgM and IgA did not show any significant change. In contrast, these immunoglobulins were not elevated in NTG or ocular hypertension. There was a higher incidence of ANA in cases of glaucoma and OHT than the control, and again there was no clear correlation with severity of the glaucoma. Although these results suggested an association of raised levels of $\operatorname{IgG}$ and higher incidence of ANA in patients with glaucoma, the evidence was insufficient to suggest that disorders of the immune system have an aetiological significance in POAG.

Wax et al looked also at differences in subpopulations of lymphocytes in patients with NTG and high-pressure glaucoma and compared them with an age-matched 
control population. Patients with NTG had a higher percentage of lymphocytes that contained the lymphocyte antigens CD8 and HLA in comparison with their POAG or control counterparts. Lymphocytes that contain these antigens are a subset of cytotoxic or 'killer' $\mathrm{T}$ cells thought to be particularly important components of the cellular immune system response to certain pathogens that have invaded the target and have been ingested by it. In addition, they found that NTG patients had elevated serum levels of cytokines such as interleukin-10, a multifunctional cytokine with both immunosuppressive or immunostimulatory effects. ${ }^{20}$

It has also been suggested that serum autoantibodies that crossreact with GAGs around the optic nerve may play a significant role in the development of NTG in some patients with systemic autoimmune diseases. ${ }^{11} \mathrm{~A}$ recent study showed ${ }^{21}$ that the immune system plays a key role in an animal's ability to resist the damaging consequences of an increase in IOP. Rats deprived of $\mathrm{T}$ cells were found to be more prone to IOP-induced retinal ganglion cell (RGC) loss than rats with normal T-cell populations. The ability of rats to resist IOPinduced RGC death can be improved by vaccination with antigens residing in the eye to protect RGCs against IOPinduced-death. These studies suggested that the onset and prognosis of glaucoma might be decided by two sets of genetic factors: those that determine susceptibility to disease development and immune factors which may determine progression.

These results support the hypothesis that humoral immune mechanisms may have a role in the pathogenesis of NTG. However, this study has shown that IgA levels are increased but ENA levels are normal, unlike previous studies. This apparent difference may be owing to geographical or racial differences in the distribution of these markers, so perhaps multi-centre studies with much larger groups of patients need to be undertaken.

If autoantibodies and paraproteins are elevated in NTG, there are implications for basic science research and for the clinical management of patients. If patients with NTG have a higher incidence of autoimmune markers, it would add support to the search for autoantigens involved in the pathogenesis of this disease. It would also raise the issue of whether patients with NTG should be screened for autoimmune disease and whether patients with known autoimmune disease should be screened for NTG, because this is a condition often diagnosed late when irreversible field loss has already occurred.

Although glaucomatous optic nerve degeneration is a leading cause of worldwide blindness, the precise cellular mechanisms underlying neurodegeneration in glaucoma are not clear. It is vital that research continues to elucidate the potential role of the immune system in glaucomatous neurodegeneration and the possibility of alternative modalities of treatment.

\section{References}

1 Halpern DL, Grosskreutz CL. Glaucomatous optic neuropathy: mechanisms of disease. Ophthalmol Clin North Am 2002; 15(1): 61-68.

2 Klein BEK, Klein R, Sponsel WE, Franke T, Cantor LB, Martone J et al Prevalence of glaucoma: the Beaver Dam Eye Study. Ophthalmology 1992; 99: 1499-1504.

3 Kamal DS, Hitchings RA. Normal tension glaucoma-a practical approach. Br J Ophthalmol 1998; 82: 835-840.

4 The collaborative normal-tension glaucoma study group. The effectiveness of IOP reduction in the treatment of normal tension glaucoma. Am J Ophthalmol 1998; 126: 498-505.

5 Butt Z, McKillop G, O'Brien C, Allan P, Aspinall P. Measurement of ocular blood flow velocity using colour Doppler imaging in low tension glaucoma. Eye 1995; 9: 29-33.

6 Hayreh SS, Zimmerman MB, Podhajsky P, Alward WL. Nocturnal arterial hypotension and its role in optic nerve head and ocular ischemic disorders. Am J Ophthalmol 1994; 117: 603-624.

7 Meyer JH, Brandi-Dohrn J, Funk J. Twenty four hours blood pressure monitoring in normal tension glaucoma. $\mathrm{Br} J$ Ophthalmol 1996; 80: 864-867.

8 O'Brien C, Butt Z, Ludham C, Detkova P. Activation of the coagulation cascade in untreated primary open angle glaucoma. Ophthalmology 1997; 104: 725-730.

9 Cartwright MJ, Grajewski AL, Friedberg ML, Anderson DR, Richards DW. Immune-related disease and normal-tension glaucoma. A case-control study. Arch Ophthalmol 1992; 110: 500-502.

10 Wax MB, Tezel G, Edward PD. Clinical and histpathological findings of a patient with normal pressure glaucoma. Arch Ophthalmol 1998; 116: 993-1001.

11 Tezel G, Edward DP, Wax MB. Autoantibodies to optic nerve head glycosaminoglycans in patients with glaucoma. Arch Ophthalmol 1999; 117: 917-924.

12 Maruyama I, Obguro H, Ikeda Y. Retinal ganglion cells recognized by serum autoantibody against gamma-enolase found in glaucoma patients. Invest Ophthalmol Vis Sci 2000; 41(7): 1657-1665.

13 Romano C, Barrett DA, Li Z, Pestronk A, Wax MB. Antirhodopsin antibodies in sera from patients with normal pressure glaucoma. Invest Ophthalmol Vis Sci 1995; 36(10): 1968-1975.

14 Wax MB, Tezel G, Saito I, Gupta RS, Harley JB, Li Z et al. Anti-Ro/SS-A positivity and heat shock protein antibodies in patients with normal pressure glaucoma. Am J Ophthalmol 1998; 125: 145-157.

15 Yang J, Tezel G, Patil RV, Romano C, Wax MB. Serum autoantibody against glutathione $S$-transferase in patients with glaucoma. Invest Ophthalmol Vis Sci 2001; 42(6): 1273-1276.

16 O'Connell TX, Horita TJ, Barsam K. Understanding and interpreting serum protein electrophoresis. Am Fam Physician 2005; 71(1): 105-112.

17 Wax MB, Barrett DA, Pestronk A. Increased incidence of paraproteinemia and autoantibidies in patients with 
normal-pressure glaucoma. Am J Ophthalmol 1994; 117(5): 561-568.

18 Kyle RA. Diagnostic criteria of multiple myeloma. Hematol Oncol Clin North Am 1992; 6: 347-369.

19 Nagasubramanian S, Rahi AHS, Gloster J. Immunological investigations in chronic simple glaucoma. Trans Ophthal Soc (UK) 1978; 98: 22-27.
20 Wax MB, Yang J, Han Z, Patil RV. Comparison of activated $\mathrm{T}$ cell subsets and serum cytokine levels in patients with glaucoma. Invest Ophthalmol Vis Sci 1999; 40: S566.

21 Bakalash S, Kipnis J, Yoles E, Schwartz M. Resistance of retinal ganglion cells to an increase in intraocular pressure is immune-dependent. Invest Ophthalmol Vis Sci 2002; 43(8): 2648-2653. 